\title{
Parameterized Electromechanical Model for Magnetic Bearings with Induced Currents *
}

\author{
Virginie KLUYSKENS** and Bruno DEHEZ** \\ **Universite Catholique de Louvain, Center for Research in Mechatronics \\ Place du Levant, 3, 1348 Louvain-la-Neuve, Belgium \\ E-mail: virginie.kluyskens@uclouvain.be,bruno.dehez@uclouvain.be
}

\begin{abstract}
The purpose of this paper is to present a parameterized electromechanical model taking into account both the electromagnetic nature of the forces acting inside a magnetic bearing and the general rotating machinery aspects, as well as their interactions. This model specifically includes the effect of induced currents. These induced currents in the bearings are submitted to inductive and resistive effects, as well as skin effect, and the influence of these effects is considered. It is shown that the stability of a bearing may be analyzed, based on this model. The parameters of the model are identified from experimental results for an existing semi-passive magnetic bearing, and it is shown that the forces predicted by the model are close to experimental measurements.
\end{abstract}

Key words: Induced Currents, Magnetic Bearing, Electromechanical Model, Unstable Behavior, Skin Effect

\section{Introduction}

Magnetic bearings can prove to be useful in various situations, like high-speed or vacuum applications where classical roller bearings reach their limits. These bearings can be classified into different categories: active, passive or semi-passive.

A good compromise between low cost and high performance can be achieved via a hybrid magnetic bearing ${ }^{(1)}$, some degrees of freedom being controlled, others not.

However, studies have shown the onset of unexpected vibrations on semi-passive magnetic bearings ${ }^{(2)}$. These are unexpected because they take place after the critical speeds of the system have been passed, and they are not due to gyroscopic effects. To understand this phenomenon, we developed in Ref. (3) an electromechanical model based on the idea that the forces due to the interaction between the induced currents and the magnetic fields can be modeled by introducing damping into the mechanical equations governing the magnetic bearing motion. This model, whose main features are presented in this paper, had already been validated in Ref. (3) on the basis of results obtained using a finite element software on a simplified case modeled in $3 \mathrm{D}$, and on experimental results for this simplified case.

The first objective of this electromechanical model was to fully understand and explain the observed unstable behavior. The second objective is now to have a practical tool in order to design new stable magnetic bearings, or possibly to correct existing unstable magnetic bearings. With this model, this can be done on the basis of a few experimental measurements, or on the basis of a finite element model of the bearing. Similar models have already been developed for particular fully passive electrodynamic bearings ${ }^{(4),(5)}$, but here a macroscopic point of view is chosen as to the electromagnetic phenomena involved in the system, which generalizes the model to any kind of magnetic bearing. Furthermore, 
there is no need to develop an often complex analytical solution for the magnetic and electric fields.

The objective of this paper is to present some refinements made on the previous electromechanical model ${ }^{(3)}$ : the damping coefficient representing the forces due to induced currents is no longer considered constant, but it depends on the inductive and the resistive behavior of the conducting part where the induced currents occur. Furthermore, the skin effect is taken into account. And finally, a more advanced analysis of the stability conditions is made. It is shown that the model predicts stable and unstable speed ranges. This electromechanical model is validated on an existing semi-passive magnetic bearing (Fig.1).

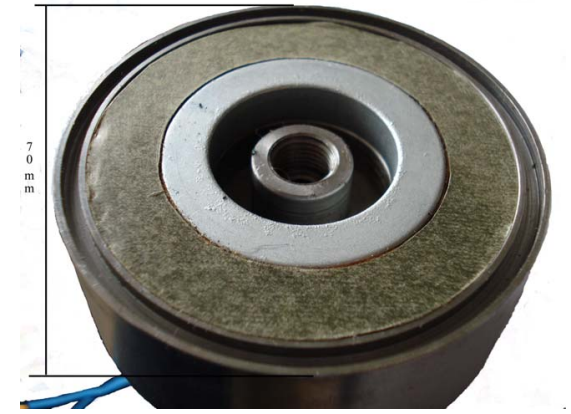

Figure 1: Stator of semi-passive magnetic bearing

\section{Model}

Magnetic bearings are electromechanical systems with a strong interaction between the electromagnetic and mechanical aspects. Therefore it is important to take both aspects into account when modeling the behavior of a magnetic bearing.

First, a short general reminder of the mechanical equations is made, showing that damping associated with rotating parts can generate an unstable behavior. Secondly, an electromechanical model is developed integrating both the rotating model and the electromagnetic nature of the forces acting on a magnetic bearing.

\subsection{Mechanical approach}

When writing the mechanical equations of a system with a rotating shaft submitted to elastic and damping forces, it is important to look at where the damping occurs ${ }^{(6)}$.

Let us define:

$c_{r}$, the damping coefficient associated with rotating parts of the system;

$c_{n r}$, the damping coefficient associated with non-rotating parts of the system;

$k$, the stiffness coefficient of the elastic force;

$x, y$, the position of the rotor in the plane perpendicular to the rotation axis;

$z=x+j y$, a complex notation used to express the position of the rotor and to write the equations of motion.

The equation of motion of a point mass, $\mathrm{m}$, attached to a massless shaft is then:

$$
m \ddot{z}+\left(c_{n r}+c_{r}\right) \dot{z}+\left(k-j \omega c_{r}\right) z=0
$$

with $\omega$ the rotational speed.

The solution of this equation can be written in the form:

$$
\left\{\begin{array}{l}
x=z 0 \exp \left(-\lambda_{I} t\right) \cos \left(\lambda_{R t}\right) \\
y=z 0 \exp \left(-\lambda_{I} t\right) \sin \left(\lambda_{R t}\right)
\end{array},\right.
$$


where $\lambda_{R}$ and $\lambda_{I}$ are the real and imaginary parts of $\lambda$ :

$$
\begin{array}{r}
\lambda=\mp \frac{1}{\sqrt{2}} \sqrt{\Gamma+\sqrt{\Gamma^{2}+\left(\frac{\omega c_{r}}{m}\right)^{2}}}+ \\
j\left(\frac{c_{r}+c_{n r}}{2 m} \pm \frac{1}{\sqrt{2}} \sqrt{-\Gamma+\sqrt{\Gamma^{2}+\left(\frac{\omega c_{r}}{m}\right)^{2}}}\right)
\end{array}
$$

with:

$$
\Gamma=\frac{k}{m}-\frac{\left(c_{n r}+c_{r}\right)^{2}}{4 m^{2}} .
$$

The first solution corresponds to a damped backward whirl motion. It has a negative real part and a positive imaginary part.

The second solution corresponds to a forward whirl motion, it has a positive real part. It is damped only when the imaginary part of $\lambda$ is negative, that is for a spin speed $\omega$ smaller than a limit spin speed $\omega_{\text {lim }}$ given by:

$$
\omega_{\text {lim }}=\sqrt{\frac{k}{m}}\left(1+\frac{c_{n r}}{c_{r}}\right)
$$

When the spin speed $\omega$ is larger than $\omega_{\text {lim }}$, the whirl motion is no longer damped, but is amplified, and the rotor exhibits an unstable behavior. When the spin speed increases beyond $\omega_{\text {lim }}$, the rotor never returns to a stable behavior. The smaller the ratio non-rotating over rotating damping, the closer the limit spin speed is to the critical speed.

\subsection{Electromechanical approach}

The electromechanical model presented in this section is based on the idea that the electromagnetic forces acting within a magnetic bearing can be modeled using mechanical components like springs and dampers. Indeed, on the one hand, the reluctance forces can be modeled by stiffnesses, as they are proportional to the relative displacement, whereas, on the other hand, the Lorentz forces due to the interaction between induced currents, due to relative speed, and magnetic fields, can be modeled by introducing damping into the equations. Depending on the location where the eddy currents take place, they will be modeled by rotating damping (in the rotor) or by non-rotating damping (in the stator).

However, these Lorentz forces can not be modeled by simply replacing the rotating damping coefficient by an appropriate constant in Eq. (1). Indeed, when an electromotive force is induced on a conducting piece, the generated currents are subjected to inductive end resistive effects. Since the Lorentz forces are proportional to the induced currents, and act in a direction determined by these induced currents, the forces are also subjected to these resistive and inductive effects. These effects will be felt on the orientation of the force: the induced currents undergo a phase shift relative to the electromotive force function of the inductive and resistive effects. They will also have consequences on the norm of the force: the norm of these induced currents depends on the norm of the electromotive force through the impedance, and then, through the resistive and inductive effects. 
In order to quantatively formalize these effects, we will first consider that: -there are only eddy currents in the rotor;

-the resistive effects taking place in the rotor can be represented by a global resistance $R$; -the inductive effects taking place can be represented by a global inductance $L$.

Next, we will introduce, as validated in Ref. (3), a complex rotating damping coefficient, $c_{r}=c_{r}^{\prime}+j c_{r}^{\prime \prime}$, where:

-the real part, $c_{r}^{\prime}$, corresponding to ordinary damping, can be related to the active power dissipated in the global resistance of the rotor;

-the imaginary part, $c_{r}^{\prime \prime}$, can be related to the reactive power produced by the global inductance of the rotor.

In a more rigorous way, this can be written as follows:

$$
\left\{\begin{array}{l}
\omega^{2} c_{r}^{\prime} \propto e i \cos \theta \\
\omega^{2} c_{r}^{\prime \prime} \propto e i \sin \theta
\end{array}\right.
$$

where $\theta$, the phase shift between the electromotive force $e$ and the induced current $i$, is given by:

$$
\theta=\arctan (\omega L / R) .
$$

Considering the global impedance of the rotor, the relation between the induced current $i$ and the electromotive force $e$ is:

$$
i=\frac{e}{\sqrt{R^{2}+(\omega L)^{2}}} .
$$

Finally, assuming that the electromotive force $e$ is proportional to the rotational speed $\omega$, Eqs (4) to (6) lead to:

$$
\left\{\begin{array}{l}
c_{r}^{\prime}=c_{r} \cos \theta, \\
c_{r}^{\prime \prime}=c_{r} \sin \theta
\end{array}\right.
$$

with:

$$
c_{r}=c_{m} \frac{1}{\sqrt{R^{2}+(\omega L)^{2}}},
$$

and $c_{m}$ a proportionality constant.

In the particular case of a purely resistive conducting piece, where the inductance is zero, the damping coefficient is real and constant.

Finally, by substituting the complex damping coefficient into the equations of motion, we obtain:

$$
\left(\begin{array}{cc}
m & 0 \\
0 & m
\end{array}\right)\left(\begin{array}{l}
\ddot{x} \\
\ddot{y}
\end{array}\right)=\left(\begin{array}{c}
F_{X} \\
F_{Y}
\end{array}\right),
$$

with:

$$
\begin{aligned}
& \left(\begin{array}{l}
F_{X} \\
F_{Y}
\end{array}\right)=\left(\begin{array}{cc}
-c_{n r}-c_{r} \cos \theta & c_{r} \sin \theta \\
-c_{r} \sin \theta & -c_{n r}-c_{r} \cos \theta
\end{array}\right)\left(\begin{array}{l}
\dot{x} \\
\dot{y}
\end{array}\right) . \\
& +\left(\begin{array}{cc}
-k_{n r}-c_{r} \omega \sin \theta & c_{r} \omega \cos \theta \\
-c_{r} \omega \cos \theta & -k_{n r}-c_{r} \omega \sin \theta
\end{array}\right)\left(\begin{array}{l}
x \\
y
\end{array}\right)
\end{aligned}
$$


The limit spin speed is then equal to:

$$
\omega_{\text {lim }}=\left(1+\frac{c_{n r}}{c_{r} \cos \theta}\right)\left(\omega_{d y n} \pm \sqrt{\omega_{d y n}^{2}+\omega_{c r}^{2}}\right),
$$

with:

$$
\left\{\begin{array}{l}
\omega_{c r}=\sqrt{k / m} \\
\omega_{d y n}=c_{n r} \tan \theta /(2 m)
\end{array} .\right.
$$

Let us note that Eq. (10) is a non-linear implicit equation since $\theta$ and $c_{r}$ depends on $\omega$ in a non-linear way.

\section{Skin effect}

One more remark needs to be made concerning this model: the impedances $R$ and $L$ used in the electromechanical model could be considered constant if the skin effect is not predominant, but usually magnetic bearings consist of massive parts and have high speeds, hence the skin effect is not negligible. The chosen law of evolution of the impedance with frequency (spin speed in this case) is explained in Ref. (7). The principle is based on a phenomenological approach. At low frequencies, the evolution law of the impedance is insignificant because the skin depth is much higher than the characteristic dimensions of the system.

This low-frequency impedance is equal to:

$$
Z_{l f}=R_{d c}+j \omega\left(L_{0}+L_{i n t}\right),
$$

with $R_{d c}$ the direct current resistance, $L_{0}$ the inductance and $L_{i n t}$ the internal inductance.

At high frequencies, the skin effect is predominant and the frequency evolutions of the resistance and the inductance are well-known. The high-frequency impedance is equal to:

$$
Z_{h f}=R_{h f}+j \omega\left(L_{0}+L_{h f}\right)
$$

with

$$
\left\{\begin{array}{l}
R_{h f}=\alpha \sqrt{\omega} \\
L_{h f}=\alpha / \sqrt{\omega}
\end{array} .\right.
$$

At intermediate frequencies, it is assumed that the impedance is a combination of the low-frequency impedance Eq. (11) and the high frequency impedance Eq. (12) according to the following expression:

$$
Z=(1-g(\omega)) Z_{l f}+g(\omega) Z_{h f}
$$

The weighing function $g(\omega)$ is equal to:

$$
g(\omega)=2 / \pi \arctan (\gamma \omega+\beta)
$$

\section{Stability analysis}

First, let us take a look at stability in Eq. (1). As mentioned before, there is one limit spin speed Eq. (3) beyond which there is no possible stable behavior. The higher the rotating damping, the lower the limit spin speed, as illustrated in Fig. 2. It can be observed that the rotating damping, which is a non conservative force, has a destabilizing role. When the ratio $c_{n r}$ over $c_{r}$ tends towards infinity ( $c_{r}$ tends to zero), the limit spin speed also tends towards infinity. But, when $c_{r}$ is not negligible, the behavior of the rotor is unstable beyond a finite limit spin speed $\omega_{\text {lim }}$. 


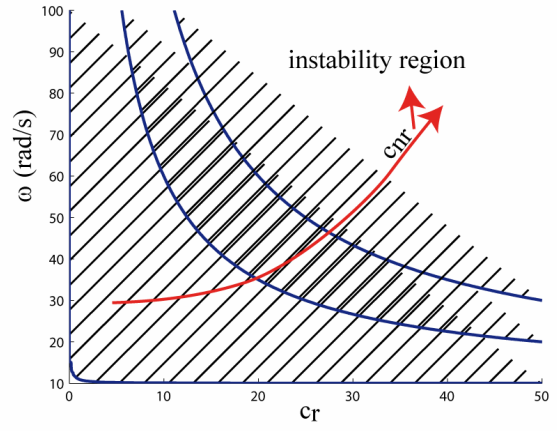

Figure 2: limit spin speed for a given set of parameters as a function of the rotating $\left(c_{r}\right)$ and the non-rotating $\left(c_{n r}\right)$ damping for the mechanical model

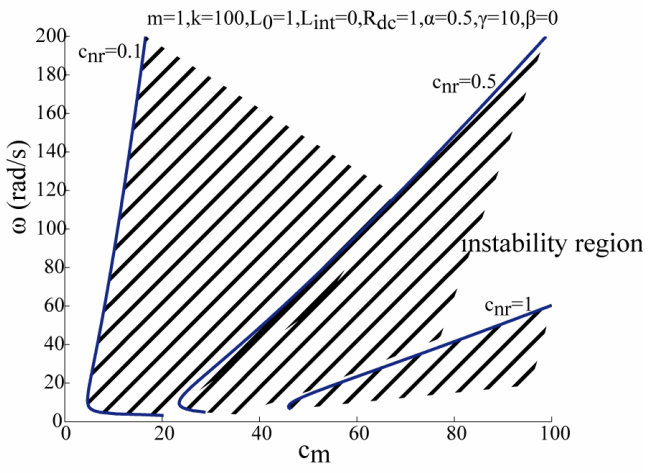

Figure 3: unstable speed range for a given set of parameters as a function of the proportionality constant of rotating damping $\left(c_{m}\right)$ and the non-rotating damping $\left(c_{n r}\right)$ for the electromechanical model

Concerning the roots of Eq (10) however, it can be seen that they give a limited speed range where the behavior of the system is unstable. Indeed, the spin speed can pass through a region where the system is unstable, and afterwards reach a region where the behavior is stable.

For instance, we can look at the instability region for a given set of parameters in Fig. 3. It is clear on this figure that the instability region has a finite limit: let us consider a rotating damping $c_{m}$ coefficient worth 10 , and a non-rotating damping $c_{n r}$ coefficient worth 0.1 , the model predicts an unstable speed range from $4.16 \mathrm{rad} / \mathrm{s}$ to $86.85 \mathrm{rad} / \mathrm{s}$. On this figure, it is also apparent that when the non-rotating damping increases, the instabilty region shrinks.

We can further analyze the equations and see that obviously inductance is favorable to stability: in Fig. 4, we see that when the inductance increases, the instability region shrinks. When the inductance is high enough, there is no longer any instability. The interpretation of Fig. 5 is more complex: the weight of low-frequency resistance decreases when the spin speed increases, and has no effect at high spin speeds.

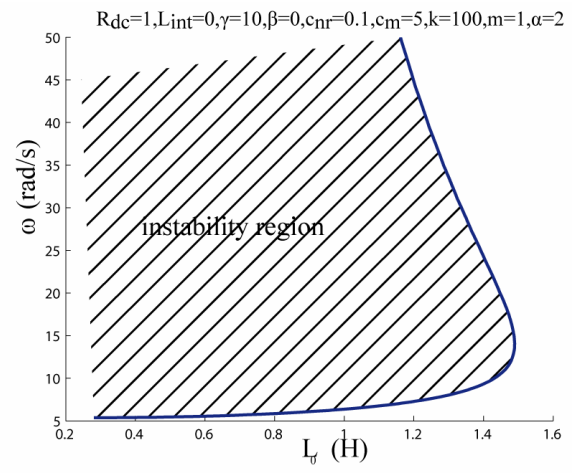

Figure 4: limit spin speed and instability region as a function of the inductance for a given set of parameters for the electromechanical model

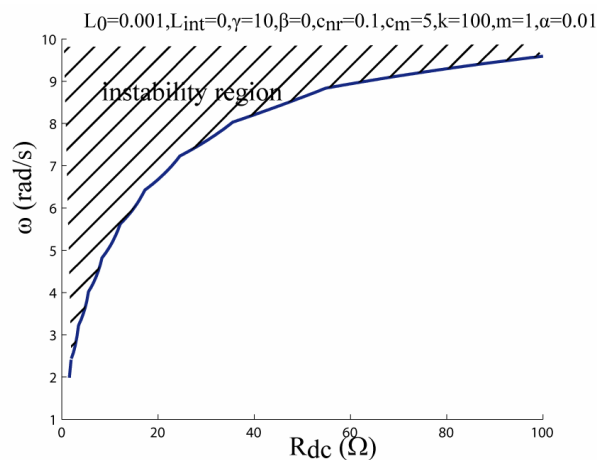

Figure 5: limit spin speed and instability region as a function of the low-frequency resistance for a given set of parameters for the electromechanical model 


\section{Application}

\subsection{Magnetic bearing}

The studied bearings (Figs. 1 and 6) were developed to be used in a flywheel energy storage system ${ }^{(2)}$. It is thus important for their energy consumption to be minimum. They are semi-passive bearings. The axial axis is controlled actively. These bearings are used in pairs: the upper bearing pulls the flywheel up, acting against gravity, and the other one

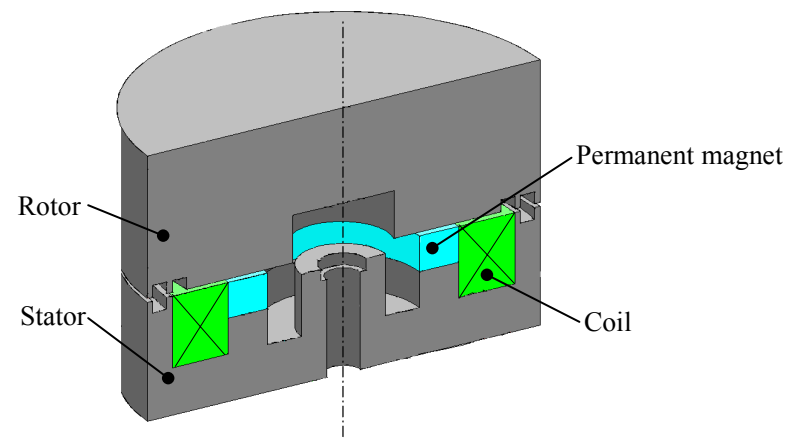

Figure 6: schematic drawing of lower semi-passive magnetic bearing

acts against the first one. The coils allow a control of the magnetic flux circulating inside the bearing, so as to stabilize the system. At equilibrium, no current circulates inside the coils, and the position of the rotor is slightly closer to the upper bearing. The passive axis is the radial axis. The radial stiffness of the bearing is obtained through reluctance forces. The teeth at the periphery of the bearing produce that reluctant force.

\subsection{Experimental setup}

The experimental setup consists of a milling machine, a force sensor with strain gauges and one magnetic bearing.

The stator of the magnetic bearing is fixed to the machine frame via the force sensor. Indeed, by the action-reaction principle, the forces acting on the rotor of the magnetic bearing are equal but opposed to the forces measured on the stator. The milling machine allows a precise positioning of the rotating part of the bearing with respect to the fixed part. The milling machine can drive the rotor of the magnetic bearing up to rotation speeds of $6300 \mathrm{rpm}$.

\section{Electromechanical model of the bearing}

\subsection{Identification}

In the electromechanical model, the parameters are unknown. First, an analysis of the physical phenomenon involved determines which parameters have to be taken into account, in the case of this semi-passive magnetic bearing. Then, the remaining parameters can be identified based on the experimental results for the forces exerted on the magnetic bearing.

First, in order to identify the stiffness coefficient intervening in the model, we measure the forces for various off-centred positions between the rotor and the stator when the rotational speed is zero. This stiffness is easily identified via a linear approximation. The average values of the stiffness in the $x$ and $y$ directions is $14024 \mathrm{~N} / \mathrm{m}$ (Fig. 7) for an air gap of $0.3 \mathrm{~mm}$. The two stifnesses are a little different $(13671 \mathrm{~N} / \mathrm{m}$, and $14378 \mathrm{~N} / \mathrm{m})$ due to a possible slight misalignment between the stator and the rotor when in centred position, 
and between the milling machine axis and the sensor axis.

Secondly, to identify the rotating damping and the impedance parameters, experiments were made with the rotor fixed in an off-centred position $(d=0.5 \mathrm{~mm})$, and the forces were measured for various spin speeds. In this case, according to the electromechanical model Eq. (9), the forces are:

$$
\begin{aligned}
& F_{x}=-\frac{c_{r}(\omega) \omega}{\sqrt{1+(\omega L / R(\omega))^{2}}} d \\
& F_{y}=-\left(k-\frac{c_{r}(\omega) \omega^{2} L / R(\omega)}{\sqrt{1+(\omega L / R(\omega))^{2}}}\right) d
\end{aligned}
$$

The stiffness force can be considered as an offset independent from the spin speed, and is subtracted from the experimental measurements. The other parameters can then be identified via the least square criterion.

The values of the parameters after identification are given in Table 1.

Let us note that in Fig. 8, $F_{x}$ is not equal to zero for a spin speed equal to zero. This is due to the possible misalignment between the stator and the rotor when in centered position, and between the milling machine axis and the sensor axis. This offset was subtracted from the force for identification purposes.

\begin{tabular}{|c|c|}
\hline$c_{m}$ & 0.32 \\
\hline$L_{0}$ & $1.147210^{-4}$ \\
\hline$L_{i n t}$ & 0.0185 \\
\hline$R_{d c}$ & $4.9310^{-11}$ \\
\hline$\alpha$ & 0.0135 \\
\hline$\gamma$ & 0.2278 \\
\hline$\beta$ & $7.742110^{-12}$ \\
\hline
\end{tabular}

Table 1: values of the parameters for the magnetic bearing

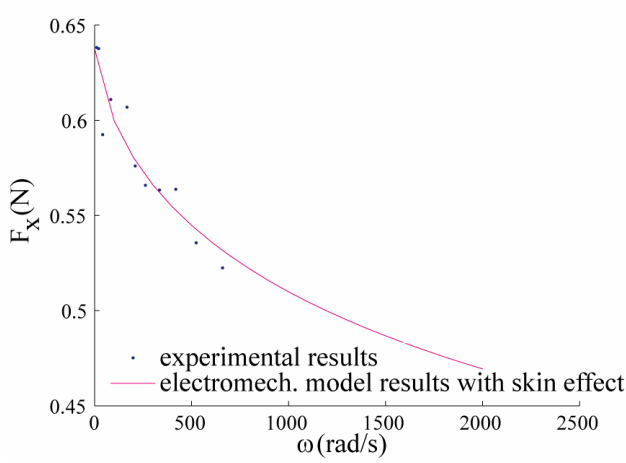

Figure 8: Experimental measurements and forces predicted by the parametrized electromechanical model for the force perpendicular to the relative displacement

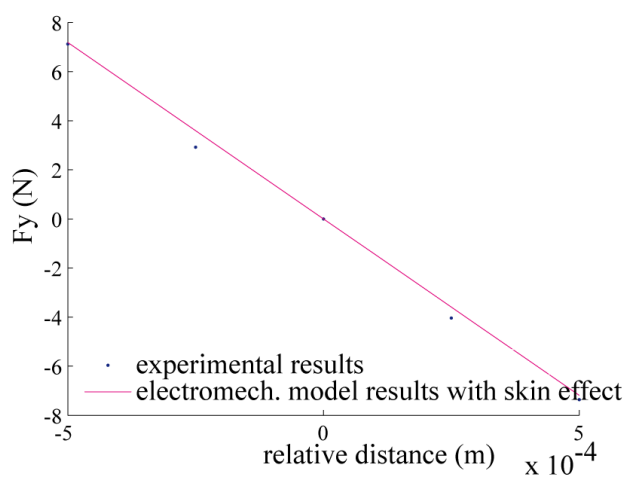

Figure 7: stiffness in the $y$ direction of the magnetic bearing (air gap of $0.3 \mathrm{~mm}$ )

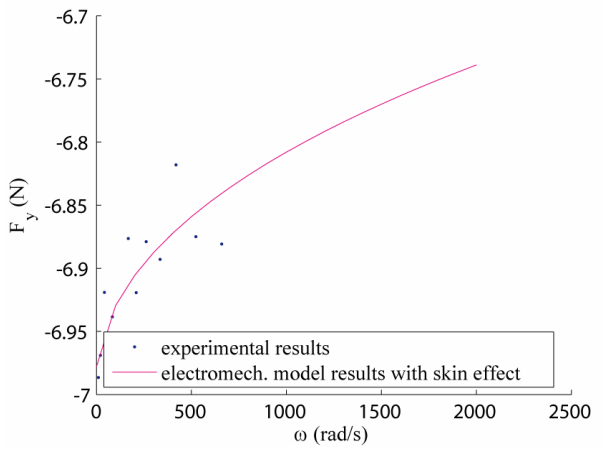

Figure 9: Experimental measurements and forces predicted by the parametrized electromechanical model for the force aligned with the relative displacement 


\subsection{Comparison}

It can be seen in Figs. 8 and 9, that parameter identification allows the electromechanical model to predict the measured forces quite well. The evolution of the forces is respected over the whole speed range, and can predict the further evolution at higher spin speeds.

These parameters were identified for an off-centred position of $0.5 \mathrm{~mm}$ between the rotor and the stator, whereas they are assumed to vary with this position according to an a priori evolution law.

\section{Conclusion}

A parametrical model has been presented representing the behavior of rotating systems submitted to electromagnetic forces, and especially forces due to the interaction of induced currents and magnetic fields. The electromagnetic forces were represented by mechanical components like springs and dampers. The inductive and resistive effects were taken into account by introducing a phase shift and a variable damping coefficient. The skin effect was also taken into account by allowing the resistive and inductive effects to vary with the spin speed. The parameters of the electromechanical model were easily identified, and showed a good agreement between the forces measured on the bearing, and the forces predicted by the electromechanical model.

This study should be completed by a finite element study of the electromagnetic bearing, but it can already be concluded that the assumptions on which the model are based are valid.

The approach proposed via this electromechanical model allows us to design or to work on existing bearings, and to predict their behavior without knowing the exact magnetic and electrical field distribution.

\section{References}

(1)Xu Yanliang, Dun Yueqin, Wang Xiuhe, Kong yu, Analysis of hybrid magnetic bearing with a permanent magnet in the rotor by FEM, IEEE Trans. on magnetics, vol 42, no4, 2007, pp1363-1366

(2)F. Faure, Magnetic suspension for flywheels (in French), Ph. D. thesis, INP Grenoble, 2003

(3)V. Kluyskens, B. Dehez, H. Ben Ahmed, Dynamical electromechanical model for magnetic bearings, IEEE Trans. on magnetics, vol 43, no7, 2007, pp3287-3292

(4)A. V. Filatov, E. H. Maslen, A method of noncontact suspension of rotating bodies using electromagnetic forces, Journal of Applied Physics, vol.91, no4, 2002, pp 2355-2371

(5)T. A. Lembke, Design and analysis of a novel low loss homopolar electrodynamic bearing, Ph. D. thesis, Royal Insitute of Technology, Sweden, 2005

(6)G. Genta, Vibration of structures and machines, $3^{\text {rd }}$ ed., New York, Springer, 1999

(7)V. Kluyskens, B. Dehez, Comparison between models predicting the evolution of the electrical impedance with frequency, submitted to International Journal of Circuit Theory and Applications 